\title{
Survival following intensive preoperative combined modality therapy with paclitaxel, cisplatin, 5-fluorouracil, and radiation in resectable esophageal carcinoma: A phase I report
}

\author{
Melvyn Goldberg, MDa \\ Jeffrey Farma, $M D^{a}$ \\ Craig Lampert, $M D^{\mathrm{b}}$ \\ Patrick Colarusso, DO \\ Lawrence Coia, MD \\ Harold Frucht, $M D^{\mathrm{d}}$ \\ Eric Goosenberg, $\mathrm{MD}^{\mathrm{d}}$ \\ Mary Beard ${ }^{\mathrm{b}}$ \\ Louis M. Weiner, MD
}

Purpose: To assess the benefits of aggressive chemoradiation therapy followed by surgery in resectable esophageal carcinoma.

Method: Twenty-nine patients with resectable carcinoma were treated with 60 Gy of radiation ( 2 Gy daily for 6 weeks) and concurrent chemotherapy consisting of continuous infusion of 5-fluorouracil $\left(200-225 \mathrm{mg} / \mathrm{m}^{2} / \mathrm{d}\right)$, paclitaxel $(25,40,50$, or $\left.60 \mathrm{mg} / \mathrm{m}^{2}\right)$ weekly over 1 hour, and cisplatin $\left(25 \mathrm{mg} / \mathrm{m}^{2}\right)$ weekly immediately following paclitaxel throughout radiation. Patients received either 4 cycles of postoperative paclitaxel $175 \mathrm{mg} / \mathrm{m}^{2}$ over 3 hours and cisplatin $75 \mathrm{mg} / \mathrm{m}^{2}$ every 3 weeks or paclitaxel $175 \mathrm{mg} / \mathrm{m}^{2}$ over 3 hours and cisplatin $75 \mathrm{mg} / \mathrm{m}^{2}$ every 3 weeks prior to the initiation of chemoradiation. After induction therapy and restaging, esophagectomy was performed 4 to 6 weeks later.

Results: Twenty-seven patients were eligible for study (26 men, 23 with adenocarcinoma). Median age was 58 years (range 30-73). The maximum tolerated dose combination was paclitaxel $50 \mathrm{mg} / \mathrm{m}^{2}$ over 1 hour weekly, cisplatin $25 \mathrm{mg} / \mathrm{m}^{2}$ over 1 hour weekly, 5 -fluorouracil $200 \mathrm{mg} / \mathrm{m}^{2} / \mathrm{d}$ by continuous infusion throughout radiotherapy and radiation to $60 \mathrm{~Gy}$. Twenty-two patients completed therapy and underwent surgical resection. Four patients had complete pathological responses and 18 had partial responses with no mortality. The commonest dose-limiting toxicity was mucositis and esophagitis $(\mathrm{n}=7)$. Median follow-up of 27 patients was 150 weeks (range 7-303). At 2-year follow-up 16/27 (59\%) were alive and 15/27 (56\%) were free of disease. At 4-year follow-up 12/27 (44\%) were alive and free of disease. Median follow-up of 22 patients undergoing esophagectomy was 205 weeks (range 26-303). At 4-year follow-up $10 / 22(45 \%)$ were alive and free of disease. For the 18 patients treated at or above the maximum tolerated dose, median follow-up was 151 weeks (range 35-206) and at 3-year follow-up 9/18 (50\%) were alive and free of disease.

Conclusion: Aggressive combined modality therapy of esophageal carcinoma was associated with excellent long-term survival in this phase I trial.

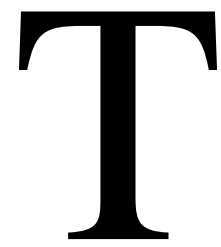

hough relatively uncommon, the incidence of carcinoma of the esophagus continues to increase. This increase is in part related to the significant increase in the number of patients presenting with adenocarcinomas secondary to gastroesophageal reflux disease and Barrett's epithelium. Approximately 12,000 Americans will develop carcinoma of the esophagus in the coming year. Overall results of surgery and radiation have been poor, and although surgery remains the standard of 
care for patients with resectable disease, median survival remains less than 10 months and those presenting with pathologic stage II (T2N0M0, T3N0M0, T1N1M0, T2N1M0) and those with stage III (T3N1M0, T4N0-1M0) disease have 5-year survivals of only $34 \%$ and $15 \%$, respectively. Several authors have demonstrated overall 5-year survivals with surgery alone ranging from $33 \%$ to $52 \% .{ }^{1-3}$ Rice and colleagues ${ }^{4}$ have identified 5-year survivals of $69 \%$ and $12 \%$ with surgery alone in patients with N0 and N1 disease, respectively, and 5-year survivals of $37 \%$ in patients who had received induction chemoradiation therapy for $\mathrm{N} 1$ disease that had been downgraded to N0 disease. Although recent randomized clinical trials have shown that preoperative chemotherapy and radiation therapy can enhance surgical outcomes, the majority of patients still relapse and die of their disease. ${ }^{5}$

A phase III trial by Herskovic and colleagues ${ }^{6}$ compared combined chemotherapy and radiation with radiation alone and showed a $26 \%$ survival advantage with combination therapy at 5 years. However, several phase II studies utilizing preoperative concurrent chemotherapy in patients with potentially resectable disease have shown variable responses with median survival ranging from 11 to 31 months and 2-year survival between $37 \%$ and $58 \% .{ }^{7,8}$ Later studies using these same protocols have failed to show any benefit with the addition of chemoradiotherapy. ${ }^{9}$ In an attempt to improve upon these results we embarked upon a protocol utilizing high-dose radiation together with an intensive chemotherapy regimen followed by surgery.

Cisplatin and 5-fluorouracil have long been recognized as having activity in esophageal carcinoma. Paclitaxel has significant antitumor activity in epidermoid carcinomas and adenocarcinomas originating in the esophagus and is the most active single agent in the treatment of metastatic esophageal cancer. ${ }^{10,11}$ Several centers have evaluated paclitaxel in esophageal cancer in fixed doses as preoperative induction combination chemotherapy with cisplatin and 5-fluorouracil in addition to chemoradiotherapy ( $45 \mathrm{~Gy}$ ) and in a phase I and II trial of induction chemotherapy at 3 dosage levels of paclitaxel with cisplatin and 5-fluorouracil and concurrent hyperfractionated radiotherapy (45 Gy to the mediastinum, 58.5 Gy to the tumor). ${ }^{12,13}$ The rationale for preoperative therapy stems in part from work done by Goldie and Coldman, ${ }^{14}$ which suggests a direct relationship between tumor burden and resistance to chemotherapy and a possible benefit to early systemic chemotherapy. This might be especially true for carcinomas of the esophagus where the lack of adventitia and a rich lymphatic supply make for early and extensive local growth and metastasis. It is possible that chemotherapeutic agents capable of functioning as a radiosensitizer as well as having activity against systemic micrometastasis could potentially result in improved survival.
In this phase I feasibility trial, escalating weekly doses of paclitaxel were administered in conjunction with daily continuous-infusion 5-fluorouracil, weekly cisplatin, and $60 \mathrm{~Gy}$ of radiation. The aim of this trial was to assess the feasibility and toxicity of such a treatment regimen and to determine the maximum tolerated dose of paclitaxel administered by 1-hour infusion when combined with 5-fluorouracil, cisplatin, and high-dose external beam radiation (60 Gy). Other objectives were to assess the pathologic complete response at surgery and to evaluate the progression-free and overall survival. The interim results of this phase I protocol have been previously reported. ${ }^{15}$ In this contribution we review the results, as well as the long-term follow-up of all patients treated on protocol.

\section{Patients and Methods \\ Eligibility Criteria}

Between January 1995 and April 1997, 29 patients were treated on protocol approved by the Institutional Review Board at Fox Chase Cancer Center. Tumors included histologically confirmed epidermoid cancer or adenocarcinoma of the esophagus, including the gastroesophageal junction. Patients required an Eastern Cooperative Oncology Group performance status of 0 to 2 and normal bone marrow, kidney, and liver function. Patients were required to have resectable disease and had to agree to proceed with surgery if disease was deemed resectable at the conclusion of combination therapy. Patients were required to have disease that was identifiable by abnormal thickening of the esophagus and nodal enlargement on computed tomography (CT) scan and by bulky disease on routine endoscopy. Esophageal ultrasonography was utilized in the minority of instances because of lack of availability to confirm the extent of intramural disease. Tis and early T1 lesions were excluded from consideration and underwent primary resection. Patients who declined this protocol were treated off protocol with induction chemoradiation utilizing cisplatin and continuous 5-fluorouracil concurrent with 45 Gy radiotherapy. All patients were required to be older than 18 years of age and were required to sign informed consent. Patients were ineligible if they (1) had received prior chemotherapy, thoracic radiotherapy, or surgical resection of the esophageal tumor; (2) had biopsy-proven invasion of the tracheobronchial tree or a tracheoesophageal fistula as demonstrated by bronchoscopy or endoscopy; or (3) had cervical malignancy.

Staging prior to induction therapy was determined by clinical assessment including CT scans of the chest, abdomen, and pelvis and bone scan. Due to the infrequent use of endoscopic esophageal ultrasound, laparoscopy, and thoracoscopy, their role in staging was not assessed. Bronchoscopy was performed routinely in upper thoracic lesions.

\section{Treatment Schema}

Radiotherapy was delivered with the aid of a 6-MeV linear accelerator in daily fractions of 2 Gy for 5 days of each week for 6 weeks to a total dose of $60 \mathrm{~Gy}$. The total dose delivered to the regional nodal areas was $40 \mathrm{~Gy}$ and the total dose delivered to the tumor was $60 \mathrm{~Gy}$. For tumors above the carina, supraclavicular and mediastinal nodes were targeted and for tumors below the carina, 
TABLE 1. Preoperative or postoperative chemotherapy dosages (eligible patients)

\begin{tabular}{lcccc}
\hline & $\begin{array}{c}\text { No. of } \\
\text { patients }\end{array}$ & $\begin{array}{c}\text { Dose of } \\
\text { paclitaxel* }^{*}\end{array}$ & $\begin{array}{c}\text { Dose of } \\
\text { cisplatin* }\end{array}$ & $\begin{array}{c}\text { Dose of } \\
\text { 5-FUt }\end{array}$ \\
\hline A & 4 & 25 & 25 & 225 \\
A & 5 & 40 & 25 & 225 \\
A & 7 & 60 & 25 & 225 \\
A & 5 & 5 & 25 & 200 \\
B & 6 & 50 & 25 & 200 \\
\hline
\end{tabular}

$A$, Postoperative adjuvant chemotherapy; $B$, preoperative combined-modality therapy; 5-FU, 5-fluorouracil.

*Concurrent with XRT; given weekly over 1 hour.

tConcurrent with XRT; given by daily intravenous infusion.

mediastinal and celiac nodes were included. Tumors of the gastroesophageal junction required treatment to the celiac nodes and mediastinum. The cone down volume included all grossly involved sites and included the primary tumor and grossly involved lymph nodes with a $2.5-\mathrm{cm}$ margin. The total dose to the spinal cord should be less than $46 \mathrm{~Gy}$. The maximum allowed deviation of dose along a field was $10 \%$ and for deviation of greater than $10 \%$ an anterior tissue compensator was used.

Concurrent preoperative chemotherapy consisted of continuous infusion 5-fluorouracil and weekly cisplatin and paclitaxel administered throughout the duration of radiation therapy. 5-Fluorouracil was administered at $225 \mathrm{mg} / \mathrm{m}^{2} / \mathrm{d}$ by continuous infusion with the aid of an external pump device. Owing to toxicities the dose of 5 -fluorouracil was later amended to $200 \mathrm{mg} / \mathrm{m}^{2} / \mathrm{d}$. Paclitaxel was administered weekly over 1 hour through the duration of radiation. Successive cohorts received doses of $25,40,50$, and $60 \mathrm{mg} / \mathrm{m}^{2}$. The preoperative and postoperative doses and the escalation schema for this study are shown in Table 1. All patients were premedicated with $\mathrm{H} 1$ and $\mathrm{H} 2$ blockers, as well as steroids. Cisplatin, at a dose of $25 \mathrm{mg} / \mathrm{m}^{2}$, was administered weekly throughout radiation and immediately following paclitaxel. Patients received adequate prehydration prior to cisplatin therapy. Toxicity was scored using the common toxicity criteria. Dose-limiting toxicity was defined as a grade 3 or grade 4 condition lasting more than 1 week. Complete blood counts were done weekly. For absolute neutrophil counts of less than 1500 and platelet counts of less than 100,000 , both radiation and chemotherapy were witheld. Cisplatin therapy was discontinued for creatinine rises of greater than 2.0 $\mathrm{mg} \%$. Initially patients who were eligible for surgery were to receive 4 cycles of postoperative adjuvant chemotherapy. This took the form of paclitaxel $\left(175 \mathrm{mg} / \mathrm{m}^{2}\right.$ over 3 hours $)$ and cisplatin $\left(75 \mathrm{mg} / \mathrm{m}^{2}\right)$ administered every 3 weeks for a total of 4 cycles. Initially patients received postoperative chemotherapy relating to the originally proscribed protocol. Although the majority of surgical patients $(15 / 22)$ received adjuvant therapy, only 3 of the patients tolerated more than 1 cycle of adjuvant therapy. Therefore the protocol was amended to 2 cycles of upfront therapy in the form of paclitaxel $\left(175 \mathrm{mg} / \mathrm{m}^{2}\right.$ over 3 hours) and cisplatin (75 $\mathrm{mg} / \mathrm{m}^{2}$ ) every 3 weeks. This was administered prior to initiation of combined chemoradiation therapy in the remaining 7 patients (Figure 1).

Following the completion of combined chemoradiation patients underwent repeat staging evaluation with CT scans of the chest, abdomen, and pelvis and barium swallow and upper gastrointestinal endoscopy. If resectable, surgery was carried out 4 to 6 weeks after combination therapy. The interval from randomization to operation ranged between 10.4 and 16.3 weeks (average 12.6 weeks, median 12.3 weeks). Total or near total esophagectomy was undertaken by 1 of the following procedures (1) Ivor Lewis esophagectomy with high intrathoracic anastomosis above the level of the azygous vein; (2) left thoracoabdominal, subtotal thoracic esophagectomy; (3) complete thoracic esophagectomy with gastric interposition and a cervical anastomosis; (4) transhiatal esophagectomy for lesions below the carina. Negative, proximal, distal, and radial margins were obtained microscopically in all patients. Abdominal lymphadenectomy included both gastroduodenal and celiac lymph nodes. A mediastinal lymphadenectomy was included with patients undergoing thoracotomy, and with transhiatal resections, an attempt was made to remove all mediastinal lymph nodes to the level of the carina.

\section{Assessment of Response and Follow-up}

Clinical complete response was the complete disappearance of all clinically apparent disease. Clinical partial response referred to a greater than or equal to $50 \%$ decrease in tumor size without the appearance of new areas of malignant disease for at least 4 weeks.

Surgical specimens were pathologically assessed for response at multiple levels longitudinally and circumferentially. Margins and lymph nodes were assessed with respect to both the gross and microscopic presence of carcinoma.

Following surgery, patients were seen in follow-up every 3 months for 2 years and thereafter every 6 months. CT scans of the chest, abdomen, and pelvis were obtained every 6 months or as clinically indicated. Barium swallow was repeated at 6 months and then as clinically indicated.

\section{Statistical Considerations}

Survival and time to progression were measured from initiation of therapy. Estimates of survival and time to progression were derived by the Kaplan-Meier method. ${ }^{16}$

\section{Results}

Between 1995 and 1997, 29 patients were treated on protocol. Two patients with stage IV disease at presentation agreed to receive the combined modality phase of the study treatment to provide additional information with respect to its tolerability. Twenty-seven patients were deemed potentially resectable with stage I-III disease and the remainder of this analysis will focus on these 27 patients. Of the 27 patients, 26 were men and 1 was a woman. Twenty-three patients had adenocarcinomas, 3 patients had squamous carcinomas, and 1 had an adenosquamous carcinoma. Eight patients had an Eastern Cooperative Oncology Group performance status of 0 and 17 patients had a performance status of 1 . The median age at diagnosis was 58 years (range 30-73). The maximal tolerated dose combination was paclitaxel $50 \mathrm{mg} / \mathrm{m}^{2}$ over 1 hour weekly, cisplatin $25 \mathrm{mg} / \mathrm{m}^{2}$ over 1 hour weekly, 5-fluorouracil $200 \mathrm{mg} / \mathrm{m}^{2} / \mathrm{d}$ by continuous infusion through the duration of radiation therapy, and 


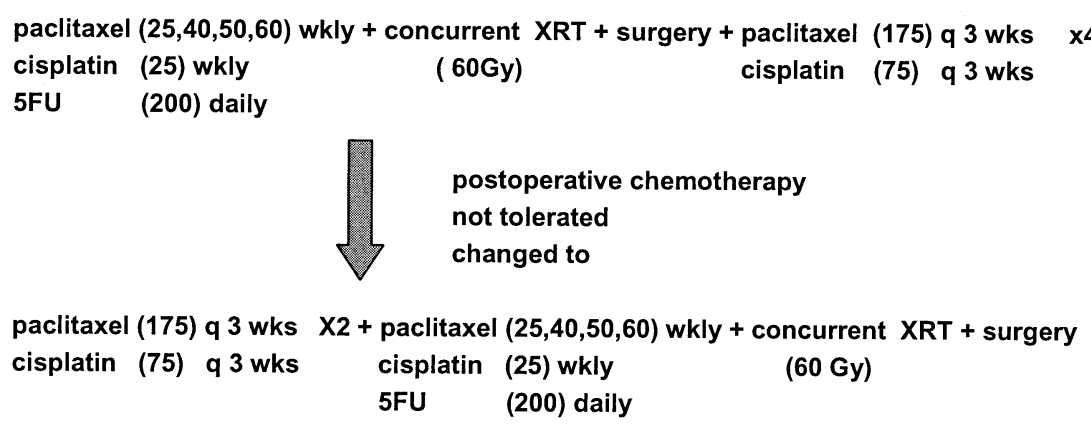

Figure 1. Treatment scheme.

radiation to $60 \mathrm{~Gy}$. Twenty-two of 27 patients completed therapy and underwent surgical resection of disease (20 adenocarcinoma, 1 squamous carcinoma, 1 adenosquamous carcinoma). Of the 5 patients who did not undergo surgical resection, 1 patient died as a result of sepsis at the conclusion of therapy, 1 had progressive disease following completion of therapy, 2 patients refused, and 1 patient had worsening of preoperative lung function that precluded surgery. Four of these patients were appointed to the protocol that required postoperative chemotherapy. Paclitaxel was given in the doses of $25 \mathrm{mg}(\mathrm{n}=1), 40 \mathrm{mg}(\mathrm{n}=1), 50 \mathrm{mg}$ $(\mathrm{n}=1)$, and $60 \mathrm{mg}(\mathrm{n}=1)$ prior to operative eligibility. Only the fifth patient was appointed to the final protocol that eliminated adjuvant therapy and received a dose of paclitaxel $(175 \mathrm{mg})$ with cisplatin as induction to combined chemoradiation therapy (paclitaxel $50 \mathrm{mg}$ ). Of the 22 patients who went to surgery there were 4 complete pathological responses (18\%). Of 11 patients who were pretreated at the maximal tolerated dose (MTD), there were 3 complete pathological responses $(27 \%)$ and 8 incomplete responses $(73 \%)$. There were no postoperative mortalities.

Dose-limiting (World Health Organization grades 3 and 4) toxicities included mucositis (7 patients), esophagitis (7 patients), diarrhea (4 patients), nausea and vomiting (3 patients), neuropathy (3 patients), line infection (3 patients), and electrolyte wasting (2 patients).

Curative resections were performed on 22 patients: thoracoabdominal esophagectomy in 1 patient, Ivor Lewis esophagogastrectomy in 5 patients, and transhiatal esophagectomy in 16 patients. Lymphadenectomy averaged 12.7 nodes per operative procedure with a range of 5 to 24 . Nine patients had a jejunostomy placed at initial thorascopic staging and 13 at operative resection. Hospitalization postoperatively averaged 15.5 days. The average blood loss per operative procedure was $425 \mathrm{~mL}$.

Postoperative complications were frequent, the most common being fever $(n=14)$, anemia $(n=13)$, wound infection $(n=6)$, bacteremia $(n=5)$, pneumonia $(n=4)$, and respiratory failure requiring intubation $(n=4)$ (Table 2).
TABLE 2. Postoperative complications $(\mathrm{n}=\mathbf{2 2})$

\begin{tabular}{lc}
\hline & Number \\
\hline Fever $\left(>100.6^{\circ} \mathrm{F}\right)$ & 14 \\
Anemia requiring transfusion(s) & 13 \\
Wound infection & 6 \\
Bacteremia & 5 \\
Respiratory failure requiring intubation & 4 \\
Pneumonia & 4 \\
Urinary tract infection & 3 \\
Anastomotic leak & 3 \\
Arrhythmia & 3 \\
Postoperative ileus & 3 \\
Pleural effusion requiring thoracentesis & 2 \\
Diarrhea & 2 \\
Deep vein thrombosis & 2 \\
Psychosis & 2 \\
Depression (severe) & 2 \\
Others & \\
Chylothorax & 1 \\
Recurrent laryngeal nerve injury & 1 \\
Intraoperative splenectomy & 1 \\
Renal failure & 1 \\
\hline
\end{tabular}

Preoperative stages of patients who underwent surgery identified T1 N0 $(n=1)$, T2 N0 $(n=14)$, T3 N0 $(n=2)$, T2 N1 $(n=2)$, and T3 N1 $(n=3)$. In patients with either no change or a decrease in stage after induction chemoradiation therapy $(\mathrm{n}=12), 8$ are alive and free of disease and 4 are dead. In those with an increase in stage $(n=10), 2$ are alive and free of disease and 8 are dead.

\section{Follow-up}

For all potentially resectable patients $(\mathrm{n}=27)$ median follow-up was 150 weeks (range 7-303). At a minimum follow-up of 2 years 16/27 (59\%) patients were alive and $15 / 27(56 \%)$ were free of disease. At a minimum follow-up of 3 years, 14/27 (52\%) patients were alive and free of disease. At a minimum follow-up of 4 years, 12/27 (44\%) patients were alive and free of disease.

Further subgroup analysis was undertaken on the 22 patients who went on to esophageal resection, as well as the 


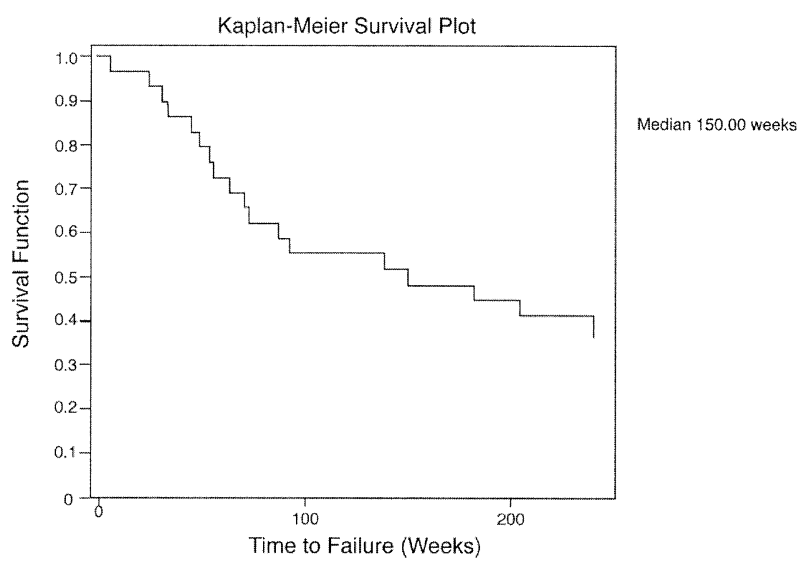

Figure 2. Actuarial survival.

16 patients who were treated at or above maximal tolerated dose. Analysis of the 22 patients who underwent esophagectomy reveals that median follow-up was 205 weeks (range 26-303). At a minimum follow-up of 2 years 12 of 22 $(55 \%)$ patients were alive and free of disease. At a minimum follow-up of 3 years 11 of $22(50 \%)$ patients were alive and free of disease. At a minimum follow-up of 4 years, 10 of 22 (45\%) patients were alive and free of disease. For the 18 patients treated at or above maximal tolerated dose, median follow-up was 151 weeks (range 35-206). Overall median survival and overall median disease-free survival has not been reached. At a minimum follow-up of 2 years, 11 of 18 patients were alive and 10 of 18 were free from disease, and at a minimum follow-up of 3 years 9 of 18 were alive and free from disease progression. Of note, 1 patient who did not proceed to surgery due to progressive disease (tracheal involvement) remains alive and free of disease at 3 years and 9 months. Of patients with progressive disease 2 patients recurred locally and 10 progressed with distant metastatic disease.

More frequent complete responses were identified in patients treated at or above MTD (0/7 below vs 4/15 above) but correlation with survival was insignificant.

Fifteen of 22 surgical patients received postoperative chemotherapy. Because of intolerance to 2 complete cycles, the adjuvant chemotherapy was discontinued and placed as induction therapy prior to chemoradiation in the last 7 of 22 patients. Kaplan-Meier actuarial survival at 4 years was 42\% (Figure 2).

\section{Discussion}

In this report we have presented the results of a phase I trial aimed at assessing the feasibility of administering an aggressive preoperative combined therapy regimen in patients with potentially operable carcinoma of the esophagus. The results of this phase I study suggest that in a select group of patients this regimen could be administered safely. Al- though toxicities were encountered, the majority of patients went on to surgery and although there were significant postoperative complications, no patients died in the perioperative setting.

The role of adjuvant chemotherapy in the setting of potentially operable disease remains controversial. Initially our patients were treated with adjuvant cisplatin and paclitaxel following the completion of concurrent chemoradiation and surgery. As few were able to tolerate the adjuvant regimen it was subsequently moved into the neoadjuvant setting. This meant that not only were patients able to receive the full regimen but more importantly it did not prevent any patient from completing the remainder of the protocol.

The National Comprehensive Cancer Network guidelines identify the use of esophageal ultrasonography and positron-emission tomography (PET) scanning as an option in the staging of esophageal carcinoma. Throughout most of this study these 2 investigative procedures were not readily available to our patients for staging purposes. Prior to an induction therapy program, patients must be staged as efficiently as possible to accurately assess response to therapy at the time of the operative procedure. We highly recommend that esophageal ultrasonography and PET scan be included in the assessment of all patients prior to induction therapy. They are relatively benign procedures compared with the more invasive procedures such as a mediastinoscopy, laparoscopy, and thoracoscopy, which we feel should be utilized in specific instances only when indicated.

In addition to reporting the results of the phase I trial, we have provided long-term data on patients treated on this trial. The progression-free survival and overall survival of patients treated on protocol suggest that an aggressive preoperative combined-modality therapy regimen might augment surgical outcomes in patients with potentially operable carcinoma of the esophagus. This is especially true for patients who had been downstaged and who subsequently have had curative resections.

A potential criticism relates to the relatively low rate of pathological complete responses obtained in this study. In keeping with other investigators, we chose a benchmark of $25 \%$ pathological complete response against which to measure the efficacy of the preoperative regimen. Our pathological complete response rate was $18 \%$ and we attribute this in part to our decision to fully examine the entire surgical specimen microscopically. Despite the relatively low pathological complete response rate, however, longterm follow-up on these patients suggests in impressive median overall survival and over all disease-free survival. The finding of residual disease in 18 of 22 surgical specimens suggests that surgery remains an essential component in the treatment of localized esophageal carcinoma. 
The progression-free survival and overall survival seen with this approach justify additional patient accrual at radiation doses of $45 \mathrm{~Gy}$ to determine if the regimen has lesser toxicity and comparable efficacy. Persistence of these preliminary indications of improved survival will warrant prospective phase III testing.

\section{References}

1. Hagen JA, DeMeester SR, Peters JH, Chandrasoma P, DeMeester TR. Curative resection for esophageal adenocarcinoma: analysis of 100 en bloc esophagectomies. Ann Surg. 2001;234:520-30.

2. Altorki N, Skinner D. Should en bloc esophagectomy be the standard of care for esophageal carcinoma? Ann Surg. 2001;234:581-7.

3. Steup WH, DeLeyn D, Deneffe G, Van Raemdonck D, Coosemans W, Lerut $\mathrm{T}$. Tumors of the esophagogastric junction. Long-term survival in relation to the pattern of lymph node metastasis and a critical analysis of the accuracy or inaccuracy of pTNM classification. J Thorac Cardiovasc Surg. 1996;111:85-94.

4. Rice TW, Blackstone EH, Adelstein DJ, et al. N1 esophageal carcinoma: the importance of staging and downstaging. J Thorac Cardiovasc Surg. 2001;121:454-64.

5. Walsh TN, Noonan MB, Hollywood D, Kelly A, Keeling N, Hennessy TP. A comparison of multimodal therapy and surgery for esophageal adenocarcinoma. $N$ Engl J Med. 1996;335:462-7.

6. Herskovic A, Martz K, Al-Sarraf M, et al. Combined chemotherapy and radiotherapy compared with radiotherapy alone in patients with cancer of the esophagus. N Engl J Med. 1992;326:1593-8.

7. Heitmiller RF, Forastiere AA, Kleinberg L, Zahurak M. Neoadjuvant chemoradiation followed by surgery for resectable esophageal cancer. Recent Results Cancer Res. 2000;155:97-104.

8. Forastiere AA, Orringer MB, Perez-Tamayo C, Urba SG, Zahurak M. Preoperative chemoradiation followed by transhiatal esophagectomy for carcinoma of the esophagus: final report. J Clin Oncol. 1993;11: 1118-23.

9. Chidel MA, Rice TW, Adelstein DJ, Kupelian PA, Suh JH, Becker M. Resectable esophageal carcinoma: local control with neoadjuvant chemotherapy and radiation therapy. Radiology. 1999;213:67-72.

10. Ajani JA, Ilson DH, Daugherty K, Kelsen DP. Paclitaxel in the treatment of carcinoma of the esophagus. Semin Oncol. 1995;22:3540.

11. Weiner LM. Paclitaxel in the treatment of esophageal cancer. Semin Oncol. 1999;26:106-8.

12. Ajani JA, Komaki R, Putnam JB, et al. A three-step strategy of induction chemotherapy then chemoradiation followed by surgery in patients with potentially resectable carcinoma of the esophagus or gastroesophageal junction. Cancer. 2001;92:279-86.

13. Wright CD, Wain JC, Lynch TJ, et al. Induction therapy for esophageal cancer with paclitaxel and hyperfractionated radiotherapy: a phase I and II study. J Thorac Cardiovasc Surg. 1997;114:811-5.

14. Goldie JH, Coldman AJ. Theoretical considerations regarding the early use of adjuvant chemotherapy. Recent Results Cancer Res. 1986;103: 30-5.

15. Weiner LM, Colarusso P, Goldberg M, Dresler C, Coia LR. Combined-modality therapy for esophageal cancer: phase 1 trial of escalating doses of paclitaxel in combination with cisplatin, 5-fluorouracil, and high-dose radiation before esophagectomy. Semin Oncol. 1997; 24(S19):93-5.

16. Kaplan E, Meier P. Non-parametric estimation from incomplete observations. J Am Stat Assoc. 1958;53:457-81. 